\title{
Pseudo Cauchy Riemann and Framed Manifolds with Physical Applications
}

\author{
Krishan Lal Duggal
}

(Communicated by H. Hilmi Hacısalihoğlu)

\begin{abstract}
We introduce a pseudo Cauchy Riemann(PCR)-structure defined by a real tensor field $\bar{J}$ of type $(1,1)$ of a real semi-Riemannian manifold $(\bar{M}, \bar{g})$ such that $\bar{J}^{2}=\lambda^{2} I$, where $\lambda$ is a function on $\bar{M}$. We prove that, contrary to the even dimensional CR-manifolds, a PCR-manifold is not necessarily of even dimension if $\lambda$ is every where non-zero real function on $\bar{M}$, supported by two odd dimensional examples and one physical model. The metric of PCR-manifold is not severely restricted. Then, we define a pseudo framed(PF)-manifold $(M, g)$ by a real tensor field $f$ such that $f^{3}=\lambda^{2} f$, where $T(M)$ splits into a direct sum of two subbundles, namely $i m(f)$ (with a PCRstructure) and $\operatorname{ker}(f)$, supported by some mathematical and physical examples. Finally, we study a revised version of a contact manifold, called contact PF-manifold, which is a particular case of a PF-manifold where $\operatorname{dim}(k e r(f))=1$. Contrary to the odd dimensional contact manifolds, there do exist even dimensional contact PF-manifolds. We also propose several open problems.
\end{abstract}

Keywords: Semi-Riemannian metric; pseudo Cauchy Riemann manifold; pseudo framed structure; linear operator; spacetime.

AMS Subject Classification (2020): Primary: 53C50 ; Secondary: 53C55; 53C80.

\section{Introduction}

Recall that Kähler's [13] 1933 work on the complex manifold theory developed into what is well-known as Kählerian geometry and their submanifolds. Cauchy Riemann(CR)[2], framed[20] manifolds and their submanifolds are interesting topics of Riemannian geometry with roots in complex manifold theory. Although the use of complex manifold theory has so far been very effective approach, a critical examination of the modern development indicates that the metric (say $\bar{g}$ ) of the underlined even dimensional (say $2 n$ ) real manifold is severely restricted by the root definition of Cauchy Riemann(CR) equations satisfying $J^{2}=-I$ where $J$ is a real linear operator. To explain this notice that the eigenvalues of $J$ are $i=\sqrt{-1}$ and $-i$ each one of multiplicity $n$. What we have then is $2 \mathrm{n}$ linearly independent null vectors in complex conjugate pair. None of these null vectors can be real because the eigenvalues are not real. As $J$ is real, the only possible signatures of the metric $\bar{g}$ are either $(0,2 n)$ or of type $(2 p, 2 q)$ with $p+q=n$ for positive definite or indefinite $\bar{g}$, respectively. Thus, this classical definition of CR-equations with real $J$ and an indefinite metric of an arbitrary signature is inappropriate for the geometry of real semi-Riemannian manifolds $(\bar{M}, \bar{g})$ and their submanifolds. In 1976, Flaherty [11] was the first who noticed that for a real $J$ the Lorentz metric is not compatible with the CR-equations. Since there do exist complex Lorentz transformations satisfying the CR-equations, he replaced the real $J$ by a complex valued endomorphism $\mathcal{J}=i J$ retaining the classical CR-equations $\mathcal{J}^{2}=-I$ where $(\bar{g}, \mathcal{J})$ satisfies the condition of an almost Hermitian structure of $\bar{M}$. See details in [11]. Although Flaherty's modification has been useful in bringing the rich geometry of Hermitian and Kählerian geometry as applied to spacetime geometry needed by physicists, in general, there is a need to revise CR-equations compatible with a vide variety of non-degenerate and degenerate metrics such that the known results of classical CR-geometry are retained as special sub-cases.

Since CR-manifolds are even dimensional, to accommodate odd dimensional manifolds, in 1963, Yano[20] 
introduced f-structure (also called framed structure) defined by a $(1,1)$ tensor field $f$ satisfying $f^{3}+f=0$ whose particular case is contact [3] structure of odd dimensional manifolds. However, for the metric framed manifolds (cf. Blair [4]), unfortunately, in this case also the existence of a semi-Riemannian metric has restrictions similar to the case of CR-manifolds. Therefore, there is also a need to revise framed and contact structures compatible with a variety of non-degenerate and degenerate metrics.

In this paper we suggest revised definitions, namely, pseudo Cauchy Riemann equations, briefly denoted by PCR-equations pseudo framed and contact pseudo framed structures, briefly denoted by PF and CPFstructures(see definitions (2.1)and (3.1), respectively, so that the semi-Riemannian metric of these revised structures is not severely restricted. We justify the existence of these three structures by means of several examples and physical models. The paper is organized as follows. In Section 2 we start with definition of a PCRstructure, explain how it is compatible with a variety of associated semi-Riemannian metrics while retaining all the known results of classical CR-structures. We have proved that PCR-manifolds are not necessarily evendimensional if $\lambda$ is every where non-zero real function, supported by two odd dimensional examples and a physical model of $5 D$ Friedmann-Robertson-Walker (FRW) spacetime. We also constructed two examples of even dimensional lightlike and non-degenerate PCR-manifolds. Then, in Section 3 we state revised PF and a revised version of a contact manifold, called CPF-manifold, which admits a contact PCR-distribution and explain the need for revising them. We construct a mathematical model of relating PF and PCR-manifolds and a physical model each of globally hyperbolic PF-manifolds and a globally null CPF-spacetime. We highlight that, contrary to the classical contact manifolds, revised contact PF-manifolds are not necessarily of odd dimension, supported by an even dimension example, with physical applications. This paper is expected to generate considerable further study on PCR, PF, CPF-manifolds and their submanifolds with applications.

\section{Pseudo Cauchy Riemann manifolds}

Let $\bar{M}$ be an $m$-dimensional real differential manifold. We say that a pseudo Cauchy Riemann structure on $\bar{M}$ is a real tensor field $\bar{J}$ of type $(1,1)$ at every point of $\bar{M}$ satisfying

$$
\bar{J}^{2}=\lambda^{2} I
$$

where $\lambda$ is a function on $\bar{M}$. Two sub-structures defined by (2.1) are:

(a) CR-structure [2] if $\lambda^{2}=-1$, rank of $\bar{J}=m$ and $m$ is even.

(b) Almost tangent structure [10] if $\lambda=0$, rank of $\bar{J}=m / 2$ and $m$ is even.

In this paper, we assume that $\lambda$ is nonzero. For those interested in the subcase $\lambda=0$ ((b) almost tangent structures) we recommend [10]. Also, it is important to mention that in 1956 Legrand [14] introduced the notion of $\pi$-structure defined by our equation (2.1) and he assumed that $\lambda$ is a nonzero complex constant whereas we assume in this paper that $\lambda$ is a nonzero real function. Suppose $\bar{M}$ admits a real $r$-dimensional distribution $D$. Following are three mutually exclusive cases of the causal character (see $\mathrm{O}^{\prime}$ Neill [17]) of $D$.
(a) $D$ is spacelike.
(b) $D$ is timelike.
(c) $D$ is lightlike.

If (a) or (b) holds, then, following the terminology of Newlander and Nirenberg [16] we say that a manifold $\bar{M}$ with a PCR-structure $(\bar{J}, \lambda)$ admits a realizable PCR-structure if the distribution $D$ is invariant $(\bar{J} D=D)$ with respect to $\bar{J}$ and it is involutive, that is, $[\bar{X}, \bar{Y}] \in D$ for every $\bar{X}, \bar{Y} \in D$. Then, $(\bar{M}, \bar{J}, \lambda)$ is called a PCR-manifold satisfying

$$
T \bar{M}=D \oplus D^{\perp}, \quad D \cap D^{\perp}=\{0\}, \quad \operatorname{dim}\left(D^{\perp}\right)=m-r .
$$

Note that there do exist non-realizable CR-structures for which see Penrose [18]. For the case (c) $D$ lightlike implies that $D^{\perp}$ is not orthogonal to $D$ since $D=D^{\perp}$ so $D+D^{\perp} \neq T \bar{M}$. Therefore, for lightlike $D$ we consider $\tilde{D}$, the complementary distribution of $D$ in $T \bar{M}$ and

$$
T \bar{M}=D \oplus_{\text {orth }} \tilde{D}, \quad D \cap \tilde{D}=\{0\} .
$$

Now we recall the following brief information (see details in [8][pages 30-40]) to recover a realizable PCRstructure for the lightlike case.

Assume that $\bar{g}$ is degenerate on $T \bar{M}$, that is, there exists a vector field $\xi \neq 0$ of $\Gamma(T \bar{M})$ such that $\bar{g}(\xi, \bar{X})=$ $0, \forall \bar{X} \in \Gamma(T \bar{M})$. The radical distribution of $T \bar{M}$, with respect to $\bar{g}$, is defined by

$$
\begin{aligned}
\operatorname{Rad} T \bar{M} & =\{\xi \in \operatorname{Rad}(T \bar{M}) ; \bar{g}(\xi, \bar{X})=0, \forall \bar{X} \in \Gamma((T \bar{M})\} \\
T \bar{M} & =\operatorname{Rad}(T \bar{M}) \oplus_{\text {orth }} S(T \bar{M}),
\end{aligned}
$$


where $S(T \bar{M})$ is a non-degenerate complementary (but not orthogonal) screen distribution of $\operatorname{Rad}(T \bar{M})$ in $T \bar{M}$. Suppose $\operatorname{dim}(\operatorname{Rad}(T \bar{M}))=r \geq 1$. Then, $\operatorname{dim}(S(T \bar{M}))=m-r$ and $\bar{g}$ has a constant rank $m-r$. In this case $(\bar{M}, \bar{g})$ is called an $r$-lightlike manifold. Set lightlike $D=\operatorname{Rad}(T \bar{M})$. If $D$ is invariant $(\bar{J} D=D)$ with respect to $\bar{J}$ which satisfies the structure equation (2.1) and $[\bar{X}, \bar{Y}] \in D, \forall \bar{X}, \bar{Y} \in D$, then, we recover a realizable lightlike PCR-structure with $\bar{M}$ an $r$-lightlike PCR-manifold. In this paper we only consider realizable PCR-structures for all the three cases. Now we highlight that contrary to the case of even dimensional CR manifolds following holds for a PCR-manifold:

Theorem 2.1. A PCR-manifold $\bar{M}$ with a PCR-structure $(\bar{J}, \lambda)$ is not necessarily even-dimensional if $\lambda$ is every where non-zero real function on $\bar{M}$.

Proof. Let $\operatorname{dim}(\bar{M})=m$ and let $\left(e_{1}, \cdots, e_{m}\right)$ be a basis for $T_{p} \bar{M}$ at a point $p$ of $\bar{M}$. Then, since $\bar{J}$ is linear, it is determined by $m^{2}$ quantities $\bar{J}_{i}^{j}$ where $\bar{J}\left(e_{i}\right)=\bar{J}_{i}^{j} e_{j}$ (sum over $j$ ). Operating both sides with $\bar{J}$ and using (2.1) we get

$$
\lambda^{2} e_{i}=\bar{J}^{2}\left(e_{i}\right)=\bar{J}_{i}^{j} \bar{J}_{j}^{k} e_{k} \Rightarrow \bar{J}_{i}^{j} \bar{J}_{j}^{k}=\lambda^{2} \delta_{i}^{k} .
$$

A matrix equation of this can be written as follows

$$
\operatorname{det}\left(\bar{J}^{2}\right)=(\operatorname{det} \bar{J})^{2}=\lambda^{2} \operatorname{det}\left(I_{m}\right)=\left(\lambda^{2}\right)^{m} \geq 0,
$$

where $I_{m}$ is the $m \times m$ unit matrix. Since $\lambda$ is non-zero real function, above implies that $m$ is not necessarily even-dimensional, which completes the proof.

Suppose $\bar{M}$ admits a semi-Riemannian metric $\bar{g}$. We know that the CR-structure tensor $\bar{J}$ is compatible with the metric tensor $\bar{g}$ of a CR-manifold if $\bar{g}(\bar{J} \bar{X}, \bar{J} \bar{Y})=\bar{g}(\bar{X}, \bar{Y})$, that is, $\bar{g}$ compatible with $\bar{J}$ is isometric. To revise this relation we say that $(\bar{M}, \bar{g}, \bar{J}, \lambda)$ is an almost metric PCR-manifold if for a non-zero function $\mu$

$$
\bar{g}(\bar{J} \bar{X}, \bar{J} \bar{Y})=\mu \bar{g}(\bar{X}, \bar{Y}), \quad \forall \bar{X}, \bar{Y} \in \Gamma(T \bar{M}),
$$

that is, $\bar{g}$ compatible with $\bar{J}$ is conformal or homothetic (in particular, isometric) if $\mu$ is constant (in particular, $\mu=1$ ), respectively. Following are two examples of odd dimensional PCR-manifolds, in support of Theorem 2.1.

Example 2.1. Let $\left\{\xi_{1}, \xi_{2}, \xi_{3}, \xi_{4}, \xi_{5}\right\}$ be a basis for $T_{p} \bar{M}$ at a point $p$ of a 5-dimensional semi-Riemannian manifold $(\bar{M}, \bar{g})$ endowed with a real tensor field $\bar{J}$ of type $(1,1)$ and two real non-zero functions $\lambda$ and $\mu$ on $\bar{M}$. Suppose

$$
\bar{J} \xi_{1}=\lambda \xi_{1}, \quad \bar{J} \xi_{2}=\lambda \xi_{3}, \quad \bar{J} \xi_{3}=\lambda \xi_{2}, \quad \bar{J} \xi_{4}=\xi_{5}, \quad \bar{J} \xi_{5}=\lambda^{2} \xi_{4} .
$$

Then, $\bar{J}^{2} \xi_{a}=\lambda^{2} \xi_{a}, \forall a=1,2,3,4,5$ holds. Suppose $\bar{g}\left(\xi_{1}, \xi_{1}\right)=\epsilon \sigma_{1}^{2}, \quad \bar{g}\left(\xi_{a}, \xi_{a}\right)=\sigma_{a}^{2}$ for each $a=2,3,4,5$ where each $\sigma_{a}$ is a real function on $\bar{M}$. Using the metric compatible equation (2.2) we get

$$
\begin{aligned}
& \bar{g}\left(\bar{J} \xi_{1}, \bar{J} \xi_{1}\right)=\epsilon \mu \sigma_{1}^{2}=\epsilon \lambda^{2} \sigma_{1}^{2} \Longrightarrow \mu=\lambda^{2}, \\
& \bar{g}\left(\bar{J} \xi_{2}, \bar{J} \xi_{2}\right)=\lambda^{2} \sigma_{2}^{2}=\lambda^{2} \sigma_{3}^{2}, \quad \bar{g}\left(\bar{J} \xi_{3}, \bar{J} \xi_{3}\right)=\lambda^{2} \sigma_{3}^{2}=\lambda^{2} \sigma_{2}^{2} .
\end{aligned}
$$

Cancelling $\lambda^{2}$ we get $\sigma_{3}^{2}=\sigma_{2}^{2}$. Now, for the two vectors $\left(\xi_{4}, \xi_{5}\right)$ we have

$$
\bar{g}\left(\bar{J} \xi_{4}, \bar{J} \xi_{4}\right)=\lambda^{2} \sigma_{4}^{2}=\sigma_{5}^{2}, \quad \bar{g}\left(\bar{J} \xi_{5}, \bar{J} \xi_{5}\right)=\lambda^{2} \sigma_{5}^{2}=\lambda^{4} \sigma_{4}^{2} .
$$

Cancelling $\lambda^{2}$ from the second equation we get $\sigma_{5}^{2}=\lambda^{2} \sigma_{4}^{2}$. Therefore, using this data and taking $\bar{X}=\Sigma_{a} \eta^{a}(\bar{X}) \xi_{a}$ we have

$$
\begin{aligned}
\bar{g}(\bar{X}, \bar{Y}) & =\epsilon \sigma_{1}^{2} \eta^{1}(\bar{X}) \eta^{1}(\bar{Y})+\sigma_{2}^{2}\left(\eta^{2}(\bar{X}) \eta^{2}(\bar{Y})+\eta^{3}(\bar{X}) \eta^{3}(\bar{Y})\right) \\
& +\sigma_{4}^{2}\left(\eta^{4}(\bar{X}) \eta^{4}(\bar{Y})+\lambda^{2} \eta^{5}(\bar{X}) \eta^{5}(\bar{Y})\right) \\
\bar{J} \bar{X} & =\lambda \eta^{1}(\bar{X}) \xi_{1}+\lambda \eta^{2}(\bar{X}) \xi_{3}+\lambda \eta^{3}(\bar{X}) \xi_{2} \\
& +\eta^{4}(\bar{X}) \xi_{5}+\lambda^{2} \eta^{5}(\bar{X}) \xi_{4} \\
\bar{J}^{2} \bar{X} & =\lambda^{2} \bar{X}, \quad \bar{g}(\bar{J} \bar{X}, \bar{J} \bar{Y})=\lambda^{2} \bar{g}(\bar{X}, \bar{Y}), \quad \forall \bar{X}, \bar{Y} \in \Gamma(T \bar{M}) .
\end{aligned}
$$

Thus, $(\bar{M}, \bar{g}, \bar{J}, \lambda)$ is an almost metric (Riemannian or Lorentzian according as $\epsilon$ is 1 or -1 , respectively) PCRmanifold. 
Physical Model 1. In 1944, Walker [19] introduced a spacially homogeneous $4 D$ spacetime, called RobertsonWalker(RW) spacetime, with metric

$$
d s^{2}=-d t^{2}+S^{2}(t) d \Sigma^{2},
$$

where $d \Sigma^{2}$ is the metric of a $3 D$ spacelike hypersurface $\Sigma$ with spherical symmetry and constant curvature $c=1,-1$ or 0 . With respect to a local spherical coordinate system $(r, \theta, \phi)$, this $3 D$ metric is given by

$$
d \Sigma^{2}=d r^{2}+f^{2}(r)\left(d \theta^{2}+\sin ^{2} \theta d \phi^{2}\right),
$$

where $f(r)=\sin r, \sinh r$ or $r$ according as $c=1,-1$ or 0 . Specially homogeneous property means that any point on one of these hypersurfaces is equivalent to any other point on the same hypersurface. To relate above metric with the metric of our Example 2.1 of a $5 D$ Lorentzian PCR-manifold we first recall a 2011 paper of Middleton and Stanley [15] on"Anisotropic evolution of $5 D$ Friedmann-Robertson-Walker(FRW) spacetime" with their choice of following metric

$$
d s^{2}=-d s^{2}+S^{2}(t)\left[d r^{2}+r^{2}\left(d \theta^{2}+\sin ^{2} \theta d \phi^{2}\right)\right]+\alpha^{2}(t) d w^{2},
$$

with respect to a coordinate system $(t, r, \theta, \phi, w)$, where there is an extra dimension with a scalar factor $\alpha(t)$ of extra 5 th dimension which evolves at a different rate, in general, than the $3 D$ scalar factor. This spacetime has a flat $(c=0)$, homogeneous and isotropic $3 D$ space and a flat extra dimension with coordinate $w$. These authors were able to write the FRW field equations exclusively in terms of the $3 D$ scalar factor $S(t)$ which produced a set of $4 D$ FRW field equations. Details on this paper (with some side references) are available in [15]. For a relation of Example 2.1 with above physical FRW-spacetime we first transform the metric equation of this example into following form

$$
d s^{2}=-d t^{2}+S^{2}(t)\left[d x^{2}+d y^{2}+d z^{2}\right]+\alpha^{2}(t) d w^{2},
$$

with respect to a local coordinates system $(t, x, y, z, w)$, where we set

$$
\epsilon \sigma_{1}^{2}=-1, \quad \sigma_{2}^{2}=\sigma_{4}^{2}=S^{2}(t), \quad \sigma_{2}^{2} \lambda^{2}=\alpha^{2}(t) .
$$

A further transformation of $3 D$ part into spherical symmetric coordinates establishes that our Example 2.1 is, in particular, a physical model of a $5 D$ Friedmann-Robertson-Walker(FRW) spacetime admitting a metric given by (2.4).

Let an almost metric PCR-manifold $\bar{M}$ admit a 2 -form $\Omega$ defined by

$$
\Omega(\bar{X}, \bar{Y})=\bar{g}(\bar{X}, \bar{J} \bar{Y}), \quad \forall \bar{X}, \bar{Y} \in \Gamma(T \bar{M}) .
$$

Proposition 2.1. Suppose an almost metric PCR-manifold $\bar{M}$ admits a 2 -form $\Omega$ defined by the equation (2.5). Then, the function (see equation (2.2)) $\mu=-\lambda^{2}$.

Proof. $\Omega(\bar{X}, \bar{Y})+\Omega(\bar{Y}, \bar{X})=0$ implies that $\bar{g}(\bar{X}, \bar{J} \bar{Y})+\bar{g}(\bar{Y}, \bar{J} \bar{X})=0$. Operating $\bar{J}$ to $\bar{X}$ on both sides and using (2.5) we get $\mu=-\lambda^{2}$.

Since Example 2.1 holds for $\mu=\lambda^{2}$ we now construct following examples to support the condition $\mu=-\lambda^{2}$ if $\bar{M}$ admits a 2 -form $\Omega$.

Example 2.2. Let $\left\{\xi_{1}, \xi_{2}, \xi_{3}, \xi_{4}\right\}$ be a basis for $T_{p} \bar{M}$ at a point $p$ of a 4-dimensional semi-Riemannian manifold $(\bar{M}, \bar{g})$ with $(\bar{J}, \lambda)$ as defined in Example 2.1. Take $\bar{J} \xi_{1}=\xi_{2}, \quad \bar{J} \xi_{2}=\lambda^{2} \xi_{1}, \quad \bar{J} \xi_{3}=\xi_{4}, \quad \bar{J} \xi_{4}=\lambda^{2} \xi_{3}$. Then, $\bar{J}^{2} \xi_{a}=\lambda^{2} \xi_{a}, \forall a=1,2,3,4$ holds. Suppose $\bar{g}\left(\xi_{a}, \xi_{a}\right)=\sigma_{a}^{2}$ for each $a=1,2,3,4$, where each $\sigma_{a}$ is a real function on $\bar{M}$. Assume $\bar{M}$ admits a 2 -form $\Omega$ defined by (2.5). Then, as per Proposition $2.1 \mu=-\lambda^{2}$. Using this,(2.2) and as in previous example we get $\sigma_{2}^{2}=-\lambda^{2} \sigma_{1}^{2}$ and $\sigma_{4}^{2}=-\lambda^{2} \sigma_{3}^{2}$. Taking $\bar{X}=\Sigma_{a} \eta^{a}(\bar{X}) \xi_{a}$ we have

$$
\begin{aligned}
\bar{g}(\bar{X}, \bar{Y}) & =\sigma_{1}^{2}\left(\eta^{1}(\bar{X}) \eta^{1}(\bar{Y})-\lambda^{2} \eta^{2}(\bar{X}) \eta^{2}(\bar{Y})\right) \\
& +\sigma_{3}^{2}\left(\eta^{3}(\bar{X}) \eta^{3}(\bar{Y})-\lambda^{2} \eta^{4}(\bar{X}) \eta^{4}(\bar{Y})\right) \\
\bar{J} \bar{X} & =\eta^{1}(\bar{X}) \xi_{2}+\lambda^{2} \eta^{2}(\bar{X}) \xi_{1}+\eta^{3}(\bar{X}) \xi_{4}+\lambda^{2} \eta^{4}(\bar{X}) \xi_{3}, \\
\bar{J}^{2} \bar{X} & =\lambda^{2} \bar{X}, \quad \bar{g}(\bar{J} \bar{X}, \bar{J} \bar{Y})=-\lambda^{2} \bar{g}(\bar{X}, \bar{Y}), \forall \bar{X}, \bar{Y} \in \Gamma(T \bar{M}) .
\end{aligned}
$$

Therefore, $(\bar{M}, \bar{g}, \bar{J}, \lambda)$ is a non-degenerate almost metric PCR-manifold which admits a 2 -form $\Omega$ defined by (2.5). 
NOTE 1. It follows from above example that even dimensional PCR-manifolds, which admit a 2 -form $\Omega$ and a real $\lambda$, do not admit a Riemannian metric. Perhaps we need a relation $\bar{J} \xi_{a}$ other than (2.3) for the Riemannian metric, which we do not know. Therefore, this case is left as an unsolved problem.

Example 2.3. Suppose $\left\{\xi_{1}, \xi_{2}, \xi_{3}, \xi_{4}, \xi_{5}\right\}$ is a basis for $T_{p} \bar{M}$ at a point $p$ of a 5-dimensional manifold $(\bar{M}, \bar{g})$ with a pair $(\bar{J}, \lambda)$ as defined in Example 2.1 and

$$
\bar{J} \xi_{1}=\lambda \xi_{1}, \quad \bar{J} \xi_{2}=\xi_{3}, \quad \bar{J} \xi_{3}=\lambda^{2} \xi_{2}, \quad \bar{J} \xi_{4}=\xi_{5}, \quad \bar{J} \xi_{5}=\lambda^{2} \xi_{4} .
$$

Then, $\bar{J}^{2} \xi_{a}=\lambda^{2} \xi_{a}, \forall a=1,2,3,4,5$ holds. Assume $\bar{M}$ admits a 2 -form $\Omega$ defined by (2.5). Then, as per Proposition 2.1, $\mu=-\lambda^{2}$. Using this and the metric compatible equation (2.2) we get $\bar{g}\left(\bar{J} \xi_{1}, \bar{J} \xi_{1}\right)=$ $-\lambda^{2} \bar{g}\left(\xi_{1}, \xi_{1}\right)=\lambda^{2} \bar{g}\left(\xi_{1}, \xi_{1}\right)$. This implies that $\bar{g}\left(\xi_{1}, \xi_{1}\right)=0$ so $\xi_{1}$ is a null vector. Now let $\bar{g}\left(\xi_{a}, \xi_{a}\right)=\sigma_{a}^{2}$ for each $a=2,3,4,5$ where each $\sigma_{a}$ is a real function on $\bar{M}$. Then following as in Example 2.1 and taking $\bar{X}=\Sigma_{a} \eta^{a}(\bar{X}) \xi_{a}$ we get

$$
\begin{aligned}
\bar{g}(\bar{X}, \bar{Y}) & =\sigma_{2}^{2}\left(\eta^{2}(\bar{X}) \eta^{2}(\bar{Y})-\lambda^{2} \eta^{3}(\bar{X}) \eta^{3}(\bar{Y})\right) \\
& +\sigma_{4}^{2}\left(\eta^{4}(\bar{X}) \eta^{4}(\bar{Y})-\lambda^{2} \eta^{5}(\bar{X}) \eta^{5}(\bar{Y})\right), \\
\bar{g}\left(\bar{X}, \xi_{1}\right) & =0, \quad \bar{g}(\bar{J} \bar{X}, \bar{J} \bar{Y})=-\lambda^{2} \bar{g}(\bar{X}, \bar{Y}), \quad \forall \bar{X}, \bar{Y} \in \Gamma(T \bar{M}) .
\end{aligned}
$$

Thus, as explained above $(\bar{M}, \bar{g}, \bar{J}, \lambda)$ is a 1-lightlike almost metric PCR-manifold which admits a 1-dimensional radical distribution $\operatorname{RadT} \bar{M}=\left\{\xi_{1}\right\}$ and 4-dimensional screen distribution $S(T \bar{M})=\left\{\xi_{2}, \xi_{3}, \xi_{4}, \xi_{5}\right\}$ such that

$$
\begin{aligned}
T \bar{M} & =\operatorname{Rad}(T \bar{M}) \oplus_{\text {orth }} S(T \bar{M}), \quad \operatorname{rank}(\bar{g})=4, \\
\bar{J} \bar{X} & =\lambda \eta^{1}(\bar{X}) \xi_{1}+\eta^{2}(\bar{X}) \xi_{3}+\lambda^{2} \eta^{3}(\bar{X}) \xi_{2} \\
& +\eta^{4}(\bar{X}) \xi_{5}+\lambda^{2} \eta^{5}(\bar{X}) \xi_{4} \\
\bar{J}^{2} & =\lambda^{2} I . \quad \bar{J} \operatorname{RadT} \bar{M}=\operatorname{RadT} \bar{M}, \quad[\bar{X}, \bar{Y}] \in \operatorname{RadT} \bar{M}, \quad \forall \bar{X}, \bar{Y} \in \operatorname{RadT} \bar{M} .
\end{aligned}
$$

Above odd dimensional example of lightlike case raises the following question? Are there any even dimensional lightlike PCR-manifolds which admit a 2-form $\Omega$. The answer is "YES" and we give following example.

Example 2.4. Let $\left\{\xi_{1}, \xi_{2}, \xi_{3}, \xi_{4}\right\}$ be a basis for $T_{p} \bar{M}$ at a point $p$ of a 4-dimensional manifold $(\bar{M}, \bar{g})$ with $(\bar{J}, \lambda)$ as defined in Example 2.1. Take $\bar{J} \xi_{1}=\lambda \xi_{1}, \quad \bar{J} \xi_{2}=\lambda \xi_{2}, \quad \bar{J} \xi_{3}=\xi_{4}, \quad \bar{J} \xi_{4}=\lambda^{2} \xi_{3}$. Then, $\bar{J}^{2} \xi_{a}=\lambda^{2} \xi_{a}, \forall a=1,2,3,4$ holds. Assume $\bar{M}$ admits a 2 -form $\Omega$ defined by (2.5) which implies $\mu=-\lambda^{2}$. Using this and the metric compatible equation (2.2) we get $\bar{g}\left(\bar{J} \xi_{1}, \bar{J} \xi_{1}\right)=-\lambda^{2} \bar{g}\left(\xi_{1}, \xi_{1}\right)=\lambda^{2} \bar{g}\left(\xi_{1}, \xi_{1}\right)$. This implies that $\bar{g}\left(\xi_{1}, \xi_{1}\right)=0$ so $\xi_{1}$ is a null vector. Similarly, one can show that $\xi_{2}$ is also null. Now let $\bar{g}\left(\xi_{a}, \xi_{a}\right)=\sigma_{a}^{2}$ for each $a=3,4$ where each $\sigma_{a}$ is a real function on $\bar{M}$. Proceeding as in Example 2.2 and taking $\bar{X}=\Sigma_{a} \eta^{a}(\bar{X}) \xi_{a}$ we get

$$
\begin{aligned}
& \bar{g}(\bar{X}, \bar{Y})=\sigma_{3}^{2}\left(\eta^{3}(\bar{X}) \eta^{3}(\bar{Y})-\lambda^{2} \eta^{4}(\bar{X}) \eta^{4}(\bar{Y})\right) \\
& \bar{g}\left(\bar{X}, \xi_{1}\right)=0, \quad \bar{g}\left(\bar{X}, \xi_{2}\right)=0, \quad \bar{g}(\bar{J} \bar{X}, \bar{J} \bar{Y})=-\lambda^{2} \bar{g}(\bar{X}, \bar{Y}), \quad \forall \bar{X}, \bar{Y} \in \Gamma(T \bar{M}) .
\end{aligned}
$$

Thus, $(\bar{M}, \bar{g}, \bar{J}, \lambda)$ is a 2-lightlike almost metric PCR-manifold which admits a 2-form $\Omega$ with 2-dimensional radical distribution $D=\operatorname{Rad} T \bar{M}=\left\{\xi_{1}, \xi_{2}\right\}$ and 2-dimensional screen distribution $\tilde{D}=S(T \bar{M})=\left\{\xi_{3}, \xi_{4}\right\}$ such that

$$
\begin{aligned}
T \bar{M} & =\operatorname{Rad}(T \bar{M}) \oplus_{\text {orth }} S(T \bar{M}), \quad \operatorname{rank}(\bar{g})=2, \\
\bar{J} \bar{X} & =\lambda \eta^{1}(\bar{X}) \xi_{1}+\lambda \eta^{2}(\bar{X}) \xi_{2}+\eta^{3}(\bar{X}) \xi_{4}+\lambda^{4} \eta^{3}(\bar{X}) \xi_{3} \\
\bar{J}^{2} & =\lambda^{2} I, \bar{J} \operatorname{RadT} \bar{M}=\operatorname{RadT} \bar{M}, \quad[\bar{X}, \bar{Y}] \in \operatorname{RadT} \bar{M}, \quad \forall \bar{X}, \bar{Y} \in \operatorname{RadT} \bar{M} .
\end{aligned}
$$

NOTE 2. Following Examples 2.3 and 2.4 one can show that there do exist odd and even dimensional $r$ lightlike almost metric PCR-manifolds which admit a 2 -form $\Omega$ defined by (2.5). Since lightlike manifolds are usually studied as hypersurfaces or higher co-dimensional submanifolds of semi-Riemannian manifolds, in a followup paper we plan to discuss more examples of lightlike PCR-submanifolds.

Let $\bar{\nabla}$ be Levi-Civita connection on $\bar{M}$ with 2 -form $\Omega$ as defined above. Then

$$
\left(\bar{\nabla}_{\bar{X}} \Omega\right)(\bar{Y}, \bar{Z})=\bar{g}\left(\bar{Y},\left(\bar{\nabla}_{\bar{X}} \bar{J}\right) \bar{Z}\right), \quad \forall \bar{X}, \bar{Y}, \bar{Z} \in \Gamma(T \bar{M}) .
$$

We say that a tensor field $\mathrm{N}_{\bar{J}(\lambda)}$ of type $(1,2)$ defined by

$$
N_{\bar{J}(\lambda)}(\bar{X}, \bar{Y})=[\bar{J}, \bar{J}](\bar{X}, \bar{Y})=[\bar{J} \bar{X}, \bar{J} \bar{Y}]+\lambda^{2}[\bar{X}, \bar{Y}]-\bar{J}([\bar{X}, \bar{J} \bar{Y}]+\bar{J}[\bar{J} \bar{X}, \bar{Y}]),
$$


$\forall \bar{X}, \bar{Y} \in \Gamma(T \bar{M})$, is called pseudo Nijenhuis tensor of $\bar{J}$. Using the terminology of CR-structures we say that PCR-structure is normal if $N_{\bar{J}(\lambda)}$ vanishes. Suppose an almost metric PCR-manifold $\bar{M}$ admits 2 -form $\Omega$. Then, $\bar{M}$ is called almost K manifold (briefly denoted by AK-manifold) if $\Omega$ is closed,i.e., $d(\Omega)=0$. Also, an almost metric PCR-manifold is called metric PCR-manifold if its PCR-structure is normal, that is, $N_{\bar{J}(\lambda)}$ vanishes. Then, AK-manifold will be called K-manifold.

Suppose $S(p)$ is the sectional curvature of a $K$-manifold $\bar{M}$ for a section $p$ in $T \bar{M}$ and an invariant section spanned by a non-null tangent vector $\bar{X}$ (i.e. $\bar{g}(\bar{X}, \bar{X}) \neq 0$ ) and $\bar{J} \bar{X}$. The sectional curvature of $\bar{M}$ with respect to an invariant $S(p)$ is called the invariant sectional curvature and if $\bar{X}, \bar{J} \bar{X}$ are orthonormal basis for $S(p)$, then, $S(p)=\bar{g}(\bar{R}(\bar{X}, \bar{J} \bar{X}) \bar{J} \bar{X}, \bar{X})$. A $K$-manifold is said to be a space-form if its invariant sectional curvature is independent of the choice of an invariant section at each point. A space-form with constant invariant sectional curvature $c$ is denoted by $\bar{M}(c)$ whose curvature tensor is given by

$$
\begin{aligned}
\bar{R}(\bar{X}, \bar{Y}) \bar{Z} & =\frac{c}{4}[\bar{g}(\bar{Y}, \bar{Z}) \bar{X}-\bar{g}(\bar{X}, \bar{Z}) \bar{Y}+\bar{g}(\bar{J} \bar{Y}, \bar{Z}) \bar{J} \bar{X} \\
& -\bar{g}(\bar{J} \bar{X}, \bar{Z}) \bar{J} \bar{Y}+2 \bar{g}(\bar{X}, \bar{J} \bar{Y}) \bar{J} \bar{Z}] .
\end{aligned}
$$

\section{Open problems 1.}

(a) Example 2.2 of even dimensional PCR-manifold and its Note 1 raises the following question? Do there exist any odd dimensional non-degenerate PCR-manifold which admits a 2 -form $\Omega$. Also, as stated in Note 1 , the existence of even dimensional Riemannian PCR-manifolds, with a 2-form $\Omega$, is another open problem.

(b) A. Gray and L. M. Hervella [12] have classified the almost Hermitian manifolds in 16 classes. Some well studied classes are:

Almost Kähler $\stackrel{\text { condition }}{\longrightarrow} d \Omega=0 . \quad$ Kähler $\stackrel{\text { condition }}{\longrightarrow} \bar{\nabla} \bar{J}=0$.

Nearly Kähler $\left.\stackrel{\text { condition }}{\longrightarrow}(\bar{\nabla})_{\bar{X}} \bar{J}\right) \bar{X}=0$. Almost semi Kähler $\stackrel{\text { condition }}{\longrightarrow} \delta \Omega=0$.

Semi- Kähler $\stackrel{\text { condition }}{\longrightarrow} \delta \Omega=0$. Hermitian $\stackrel{\text { condition }}{\longrightarrow} N=0$.

It is an open problem to investigate similar classification of almost metric PCR-manifolds subject to the functions $\lambda$ and $\mu$. New conditions are expected.

(c) We know that the vanishing of Nijenhuis tensor $N$ implies from a result of Newlander and Nirenberg [16] that the almost complex structure tensor $\bar{J}$ is integrable. It is an open question to find the integrability conditions of the almost PCR-structure tensor $\bar{J}$ if its pseudo Nijenhuis tensor vanishes.

(d) Although we have above specific examples of odd and even dimensional semi-Riemannian and lightlike metric PCR-manifolds, it is an open question of an arbitrary existence (or non-existence) of semi-Riemannian metric for a PCR-manifold.

Remark 2.1. To compare new notations with those of CR-manifolds, we say that if the PCR-manifold reduces to a CR-manifold $\left(\lambda^{2}=-1, \mu=1\right)$, then, the corresponding almost metric (resp. metric) manifold will be almost Hermitian (resp. Hermitian), the AK-manifold(resp. K-manifold) will be almost Kähler (resp. Kähler) manifold for which the real distribution $D=\operatorname{Re}(H+\bar{H})$ where $H$ is a holomorphic subbundle of $C T(\bar{M})$ such that $H \cap \bar{H}=\{0\}$ and $H$ is involutive, that is, $[\bar{X}, \bar{Y}] \in H$ for every $\bar{X}, \bar{Y} \in H$. Also, compared to the fixed condition ( $\bar{J}$ defines an isometry) for the almost Hermitian manifold we have choice of suitable values of the pair $(\lambda, \mu)$ for a variety of almost metric PCR-manifolds and as per Theorem 2.1 it is not restricted to an even dimension (see Examples 2.1 and 2.3).

For the special case of a Lorentzian PCR-manifold $(\bar{M}, \bar{g}, \bar{J}, \lambda)$, we suggest following two possible approaches which satisfy the compatibility condition (2.2). Those who are interested in using complex manifold theory, they can use Flaherty's [11] method by replacing the real $\bar{J}$ with a complex valued endomorphism $\mathcal{J}=i J$ retaining the classical CR-equations $\left(\lambda^{2}=-1\right)$ which satisfy the compatibility condition of an almost Hermitian structure of $\bar{M}$. However, although the use of complex manifold theory is "alive and well", it is carried out by a relatively small number of enthusiastic researchers and, in particular, the underline physics (as presented in Flaherty's Thesis [11] on applications of Hermitian and Kählerian geometry in general relativity) is of very mild interest. The more general approach comes from our new equation (2.1) of PCR-manifolds subject to the compatible condition (2.2) for semi-Riemannian (in particular, Lorentzian and lightlike) manifolds, supported by Examples 2.1-2.4 and a physical model. 


\section{Pseudo framed manifolds}

Let $M$ be an $(n=m+q)$-dimensional manifold. We say that a pseudo framed structure on $M$ is a real tensor $f$ of type $(1,1)$ at every point of $M$ satisfying

$$
f^{3}-\lambda^{2} f=0, \quad \operatorname{rank}(f)=m
$$

where $\lambda$ is a non-zero function (same as assumed in previous section) on $M$. Corresponding to two projection operators $P$ and $Q$ applied to $T M$, defined by

$$
\text { (i) } \lambda^{2} P=f^{2}, \quad \text { (ii) } \lambda^{2} Q=-f^{2}+\lambda^{2} I,
$$

where $I$ is the identity operator, there exist two complementary distributions $D$ and $\tilde{D}$ such that $\operatorname{dim}(D)=m$ and $\operatorname{dim}(\tilde{D})=q$. The following relations hold

$$
f P=P f=f, \quad f Q=Q f=0, \quad f^{2} P=\lambda^{2} P, \quad f^{2} Q=0 .
$$

Thus, we have a PCR-distribution $\left(D, \bar{J}=f / D, \bar{J}^{2}=\lambda^{2} I\right)$ and $f$ acts on $\tilde{D}$ as a null operator. Therefore, $T(M)$ splits into a direct sum of two subbundles, namely $i m(f)$ (with a PCR-structure) and $k e r(f)$. Suppose that kerf is parallelizable. Then, there exist global(or local) linearly independent $q$ vector fields $\left\{\xi_{a}\right\}$ at any point spanning $\tilde{D},(m+1 \leq a, b, \ldots \leq m+q)$, with its dual set $\left\{\eta^{a}\right\}$ such that

$$
f^{2}=\lambda^{2}\left(I-\Sigma_{a} \eta^{a} \otimes \xi_{a}\right) .
$$

In the above case, we say that $M$ is pseudo framed manifold, briefly denoted by PF-manifold with PF-structure denoted by $\left(M, f, \lambda, \eta^{a}, \xi_{a}\right)$. Following holds

$$
f \xi_{a}=0, \quad \eta^{a} \circ f=0, \quad \eta^{a}\left(\xi_{b}\right)=\delta_{b}^{a}, \quad Q(X)=\Sigma_{a} \eta^{a}(X) \xi_{a} .
$$

We say that PF-structure of $M$ is normal if its torsion tensor $T_{f(\lambda)}$ is zero i.e., if

$$
T_{f(\lambda)} \equiv N_{f(\lambda)}-\lambda^{2} d \eta^{a} \otimes \xi_{a}=0,
$$

where $N_{f(\lambda)}$ is the pseudo Nijenhuis tensor of $f$ as defined in previous section. Consider a semi-Riemannian metric $g$ on PF-manifold $M$. We say that $M$ is an almost metric PF-manifold if

$$
\begin{aligned}
g(f X, f Y) & =\mu\left(g(X, Y)+\epsilon_{a} \sigma_{a}^{2} \eta^{a}(X) \eta^{a}(Y)\right), \\
g\left(X, \xi_{a}\right) & =\epsilon_{a} \sigma_{a}^{2} \eta^{a}(X), \quad g\left(\xi_{a}, \xi_{a}\right)=\epsilon_{a} \sigma_{a}^{2}, \quad \forall X, Y \in \Gamma(T M),
\end{aligned}
$$

where $\epsilon_{a}=+1$ or -1 according as the corresponding $\xi_{a}$ is spacelike or timelike, $\mu$ (same as assumed in previous section) and $\sigma_{a}$ are non-zero real functions on $M$. An almost PF-structure is generalization of an almost PCR-structure $(q=0)$ whose sub-structure is classical f-structure if $\lambda^{2}=-1$ and $\mu=1$. If $q=1$ then we say that almost $\mathrm{PF}$-structure is an almost contact $\mathrm{PF}=$ structure, denoted by almost $\mathrm{CPF}$-structure, whose almost CPF-manifold, as per Proposition 2.1, is not necessarily of odd dimension (see some details on CPF-manifolds in next subsection). In particular, $M$ is an almost contact manifold [4] if $q=1, \lambda^{2}=-1, \mu=1$. Suppose an almost metric PF-manifold $M$ admits a 2-form $\Omega$. Then, we say that $M$ is almost PK manifold (briefly denoted by APK-manifold) if $\Omega$ is closed,i.e., $d(\Omega)=0$. Also, an almost metric PF-manifold is called metric PF-manifold if its PF-structure is normal. Then, APK-manifold will be called PK-manifold.

Following is a mathematical model relating PCR and PF manifolds.

Mathematical Model. Let $(\bar{M}, \bar{g}, \bar{J}, \lambda, \mu)$ be a $m$-dimensional almost metric PCR-manifold defined by $\bar{J}^{2}=$ $\lambda^{2} I$ and $\bar{g}(\bar{J} \bar{X}, \bar{J} \bar{Y})=\mu \bar{g}(\bar{X}, \bar{Y})$. Construct a product manifold

$$
M=\left[\left(\bar{M} \times M^{\prime}\right), g=\bar{g}+g^{\prime}\right],
$$

where $\left(M^{\prime}, g^{\prime}\right)$ is a $q$-dimensional semi-Riemannian manifold with $g^{\prime}$ its metric. Denote any vector field on $M$ by $X=\left(\bar{X}, \Sigma_{a} \eta^{a}(X) \frac{\partial}{\partial x^{a}}\right)$ with $\left(x^{a}\right), a=(1, \cdots, q)$, local coordinates on $M^{\prime}$ and $\eta^{a}(X)$ are $q$ smooth functions on $M$. Let $\xi_{a}=\left(0, \frac{\partial}{\partial x^{a}}\right)$ be $q$ vector fields tangent to $M$ and $f$ be a $(1,1)$ tensor field on $M$ defined by

$$
f(X)=f\left(\bar{X}, \Sigma_{a} \eta^{a}(X) \frac{\partial}{\partial x^{a}}\right)=(\bar{J} \bar{X}, 0)
$$


Then, it is easy to obtain $f^{3}=\lambda^{2} f, \quad f\left(\xi_{a}\right)=0, \quad \eta^{a} f=0, \quad \eta^{a}\left(\xi_{b}\right)=\delta_{b}^{a}$. Let $g\left(\xi_{a}, \xi_{a}\right)=\epsilon \sigma_{a}^{2}, \quad \forall a=(1, \cdots, q)$. Using all this we get

$$
g(f X, f Y)=\mu\left(g(X, Y)+\Sigma_{a} \sigma_{a}^{2} \epsilon_{a} \eta^{a}(X) \eta^{a}(Y)\right) .
$$

Therefore, $\left(M, g, f, \lambda, \mu, \xi_{a}\right)$ is mathematical model of almost metric PF-manifolds.

Example 3.1. Suppose $\left\{\xi_{1}, \xi_{2}, \xi_{3} ; \xi_{a}\right\}$ is an adapted basis for $T_{p} M$ at a point $p$ of a $(3+q)$-dimensional semiRiemannian manifold $(M, g)$ endowed with a real tensor field $f$ of type $(1,1)$ and two real non-zero function $\lambda$ and $\mu$ on $M$, where $(a=4,5, \cdots, 3+q)$. Let

$$
\begin{aligned}
f \xi_{1} & =\lambda \xi_{1}, \quad f \xi_{2}=\xi_{3}, \quad f \xi_{3}=\lambda^{2} \xi_{2}, \quad f \xi_{a}=0, \quad \forall(a=4,5, \cdots, 3+q), \\
g\left(\xi_{1}, \xi_{1}\right) & =\epsilon \sigma_{1}^{2}, \quad g\left(\xi_{2}, \xi_{2}\right)=\sigma_{2}^{2}, \quad g\left(\xi_{3}, \xi_{3}\right)=\sigma_{3}^{2}, \quad g\left(\xi_{a}, \xi_{a}\right)=\sigma_{a}^{2},
\end{aligned}
$$

where $\sigma_{1}, \sigma_{2}, \sigma_{3}$ and $\sigma_{a}$ are $(3+\mathrm{q})$ real functions on $M$. Using the metric compatible equation (2.2) and following as examples of Section 2, we get $\mu=\lambda^{2}$ and $\lambda^{2} \sigma_{2}^{2}=\sigma_{3}^{2}$. Taking $X=\Sigma_{a} \eta^{a}(X) \xi_{a}$ we have

$$
\begin{aligned}
g(X, Y) & =\epsilon \sigma_{1}^{2} \eta^{1}(X) \eta^{1}(Y)+\sigma_{2}^{2}\left(\eta^{2}(X) \eta^{2}(Y)+\lambda^{2} \eta^{3}(X) \eta^{3}(Y)\right), \\
& +\Sigma_{a} \sigma_{a}^{2} \eta^{a}(X) \eta^{a}(Y), \quad \forall X, Y \in \Gamma(T M), \\
f X & =\lambda \eta^{1}(X) \xi_{1}+\eta^{2}(X) \xi_{3}+\lambda^{2} \eta^{3}(X) \xi_{2}, \quad \operatorname{rank}(f)=3, \\
f^{2} X & =\lambda^{2}\left(X-\Sigma_{a} \eta^{a}(X) \xi_{a}\right), \quad f^{3} X=\lambda^{2} f X, \\
g(f X, f Y) & =\lambda^{2}\left(g(X, Y)+\Sigma_{a} \sigma_{a}^{2} \eta^{a}(X) \eta^{a}(Y)\right) .
\end{aligned}
$$

Therefore, $\left(M, g, f, \lambda, \xi_{a}\right)$ is an almost metric (Riemannian or Lorentzian according as $\epsilon$ is 1 or -1 , respectively) PF-manifold.

NOTE 3. In above example if $\sigma_{a}^{2}$ is replaced by $\epsilon_{a} \sigma_{a}^{2}$, then, one can get a variety of metric signatures of almost metric PF-manifolds, subject to restrictions stated in next open problems. Moreover, if $M$ admits a 2-form $\Omega$ defined by (2.4), then, $\mu=-\lambda^{2}$ so above example is not applicable. Therefore, we suggest as an exercise to follow Examples 2.2-2.4 for constructing examples of non-degenerate and lightlike almost metric PF-manifolds which admit a 2 -form $\Omega$.

Physical Model 2. A Lorentzian manifold $(M, g)$ is said to be globally hyperbolic if there exists a spacelike hypersurface $H$ such that every endless causal curve intersects $H$ once and only once. Such a hypersurface is called a Cauchy surface. If $M$ is globally hyperbolic, then (a) $M$ is homeomorphic to $\mathbf{R} \times H$ and for each $t$, $\{t\} \times H$ is a Cauchy surface, (b) if $H^{\prime}$ is any compact hypersurface without boundary, of $M$, then $H^{\prime}$ must be a Cauchy surface.

Although simple examples of globally hyperbolic spacetimes are Minkowski and the Einstein static universe, to include some more physically important models, such as Robertson-Walker and Schwarzschild spaces, Beem and Ehrlich [1] constructed a large extended class of globally hyperbolic Lorentz manifolds as follows:

Let $\left(M_{1}, g_{1}\right)$ and $\left(M_{2}, g_{2}\right)$ be Lorentz and Riemannian manifolds respectively. Let $h: M_{1} \rightarrow(0, \infty)$ be a $C^{\infty}$ function and $\Pi: M_{1} \times M_{2} \rightarrow M_{1}, \bar{\Pi}: M_{1} \times M_{2} \rightarrow M_{2}$ the projection maps given by $\Pi(p, q)=p$ and $\bar{\Pi}(p, q)=q$ for every $(p, q) \in M_{1} \times M_{2}$. Then, define the metric $g$ given by

$g(X, Y)=g_{1}\left(\Pi_{\star} X, \Pi_{\star} Y\right)+h(\Pi(p, q)) g_{2}\left(\bar{\Pi}_{\star} X, \bar{\Pi}_{\star} Y\right)$,

where $\Pi_{\star}$ and $\bar{\Pi}_{\star}$ are respectively tangent maps. They proved

Theorem 3.1. Let $\left(M_{1}, g_{1}\right)$ and $\left(M_{2}, g_{2}\right)$ be Lorentzian and Riemannian manifolds respectively. Then, the Lorentzian warp product manifold $M=\left(M_{1} \times_{h} M_{2}, g=g_{1} \oplus_{h} g_{2}\right)$ is globally hyperbolic if and only if both following conditions hold:

(1) $\left(M_{1}, g_{1}\right)$ is globally hyperbolic.

(2) $\left(M_{2}, g_{2}\right)$ is a complete Riemannian manifold.

For physical use of above theorem with several other examples of globally hyperbolic spacetimes (including the $5 D$ Friemann-Robertson-Walker(FRW) spacetime of Section 2) we refer Beem-Ehrlich[1] and DuggalSharma [9][pages 68-72]. Now we need following general example for the physical interpretation of a class of PF-manifolds by relating them with globally hyperbolic spacetimes.

Example 3.2. Let $\left\{\xi_{1}, \xi_{2} ; \xi_{a}\right\}$ be a basis for $T_{p} M$ at a point $p$ of a $(2+q)$-dimensional Lorentzian manifold $(M, g)$ endowed with a real tensor field $f$ of type $(1,1)$, two real non-zero functions $\lambda$ and $\mu$ on $M$. Suppose $f \xi_{1}=$ $\xi_{2}, \quad f \xi_{2}=\lambda^{2} \xi_{1}$ and $f \xi_{a}=0, \quad \forall(a=3, \cdots, 2+q)$. Suppose $g\left(\xi_{1}, \xi_{1}\right)=-1$ and $g\left(\xi_{a}, \xi_{a}\right)=\sigma_{a}^{2}, \quad \forall a=(3, \cdots, 2+$ 
q), where $\sigma_{a}$ are real functions on $M$. Assume $M$ admits a 2-form $\Omega$ defined by (2.4). Then, as per Proposition 2.1 $\mu=-\lambda^{2}$. Using this and the metric compatible equation (2.2) we get $g\left(f \xi_{1}, f \xi_{1}\right)=-\lambda^{2} g\left(\xi_{1}, \xi_{1}\right)=\lambda^{2}=g\left(\xi_{2}, \xi_{2}\right)$. Therefore, taking $g\left(\xi_{2}, \xi_{2}\right)=\lambda^{2}$ and $X=\eta^{1}(X) \xi_{1}+\eta^{2} X \xi_{2}+\Sigma_{a} \eta^{a}(X) \xi_{a}$ we have

$$
\begin{aligned}
g(X, Y) & =-\eta^{1}(X) \eta^{1}(Y)+\lambda^{2} \eta^{2}(X) \eta^{2}(Y)+\Sigma_{a} \sigma_{a}^{2} \eta^{a}(X) \eta^{a}(Y), \\
f X & =\eta^{1}(X) \xi_{2}+\lambda^{2} \eta^{2}(X) \xi_{1}, \quad \operatorname{rank}(f)=2, \\
f^{2} X & =\lambda^{2}\left(X-\Sigma_{a} \eta^{a}(X) \xi_{a}\right), \quad f^{3} X=\lambda^{2} f X, \\
g(f X, f Y) & =-\lambda^{2}\left(g(X, Y)+\sum_{a} \sigma_{a}^{2} \eta^{a}(X) \eta^{a}(Y)\right), \quad \forall X, Y \in \Gamma(T M) .
\end{aligned}
$$

Therefore, $(M, g, f, \lambda)$ is an almost metric Lorentzian PF-manifold which admits a 2-form $\Omega$ defined by (2.4).

Following are known examples of 4-dimensional globally hyperbolic spacetimes related to above example (for details see [9][pages 68-72]). First we set $q=2$ so $\operatorname{dim}(M)=4$ and let $\sigma_{3}^{2}=\lambda^{2} \alpha^{2}$ and $\sigma_{4}^{2}=\lambda^{2} \beta^{2}$, where $\alpha$ and $\beta$ are some real functions on $M$, Then, the metric (3.3) of above example takes the form

$$
g(X, Y)=-\eta^{1}(X) \eta^{1}(Y)+\lambda^{2}\left[\eta^{2}(X) \eta^{2}(Y)+\alpha^{2} \eta^{3}(X) \eta^{3}(Y)+\beta^{2} \eta^{4}(X) \eta^{4}(Y)\right] .
$$

De-Sitter spacetime. Transform the metric equation (3.4) into following form

$$
d s^{2}=-d t^{2}+a^{2} \cosh ^{2}(t / a)\left[d x^{2}+d y^{2}+d z^{2}\right],
$$

where the local basis $\left\{\xi_{1}, \xi_{2}, \xi_{3}, \xi_{4}\right\}$ is with respect to local coordinates $(t, x, y, z), \lambda=a \cosh (t / a)$ for some nonzero constant $a, \alpha^{2}=\beta^{2}=1$ and we take curvature $c=1$. Transforming $3 D$ part into spherical symmetric coordinates we get

$$
d s^{2}=-d t^{2}+a^{2} \cosh ^{2}(t / a)\left[d r^{2}+\sin ^{2} r\left(d \theta^{2}+\sin ^{2} \theta d \phi^{2}\right] .\right.
$$

Therefore, Example 3.2 is related to globally hyperbolic De-Sitter spacetime of constant curvature $c=1$ which is topologically $R^{1} \times S^{3}$ (see [9][page 69] for details).

Robertson-Walker spacetime. Recall from physical model 1 the Robertson-Walker(RW) spacetime with metric

$$
d s^{2}=-d t^{2}+S^{2}(t) d \Sigma^{2}=-d t^{2}+S^{2}(t)\left[d r^{2}+f^{2}(r)\left(d \theta^{2}+\sin ^{2} \theta d \phi^{2}\right)\right]
$$

where $d \Sigma^{2}$ is the metric of a spacelike hypersurface, $\Sigma$ has spherical symmetry and $f(r)=\sin r, \sinh r$ or $r$ according as $c=1,-1$ or 0 , with respect to a local spherical coordinate system $(r, \theta, \phi)$. The range of the coordinates is restricted from 0 to $2 \pi$ or from 0 to $\infty$ for $c=1$ or -1 respectively. For $c=1, \Sigma$ is diffeomorphic to $S^{3}$ and the corresponding RW-spacetime is globally hyperbolic, but, it is not globally hyperbolic for both cases of $c=-1,0$. See [9][pages 70-72] for details on this with references. Therefore, we consider $c=1$ for which $f(r)=\sin r$.

Replacing $\lambda^{2}=a^{2} \cosh ^{2}(t / a)$ in equation (3.5) by $S^{2}(t)$ we get the equation (3.6), with $f(r)=\sin r$, as a globally hyperbolic RW-spacetime. Therefore, Example 3.2 is related to this RW-spacetime for the case $c=1$. We leave it as an exercise to show that Friemann-Robertson-Walker(FRW) and Schwarzschild spacetimes are also physical examples of a class of almost metric Lorentzian PF-manifolds.

Physical Model 3. A lightlike manifold $(M, g)$ is called globally null manifold if it admits a single global null vector field and a complete Riemannian hypersurface. Details on this class of manifolds, with its physical use, is available in [7][Chapter 8]. Here we show that a particular case of our Example 3.2 is a physical example of globally null manifolds. Set $\lambda^{2}=1$ in the equation (3.3) and transform it into

$$
d s^{2}=-d t^{2}+d\left(x^{2}\right)^{2}+h_{a b} d x^{a} d x^{b}, \quad(a, b=3, \cdots, q),
$$

with respect to a local coordinate system $\left(x^{1}=t, x^{2}, \cdots, x^{q}\right)$. As explained in physical model 2 we assume that the PF-spacetime $(M, g)$, with $g$ its metric (3.7), is globally hyperbolic. Take two null coordinates $u$ and $v$ such that $u=t+x^{2}$ and $v=t-x^{2}$. Then, above metric transforms into

$$
d s^{2}=-d u d v+h_{a b} d x^{a} d x^{b} .
$$

The absence of $d u^{2}$ and $d v^{2}$ in above metric implies that $\{v=$ constant. $\}$ and $\{u=$ constant. $\}$ are lightlike hypersurfaces of $M$. Let ( $H, h, v=$ constant) be one of this lightlike hypesurface with 1-dimensional RadTM 
distribution $D$ generated by the null vector $\left\{\xi_{1}\right\}$ in $M$. A leaf $H^{\prime}$ of the $(m-1)$-dimensional screen distribution of $M$ is Riemannian with metric $d \Omega^{2}=h_{a b} d x^{a} d x^{b}$. In particular, there will be many global timelike vector fields in this globally hyperbolic PF-spacetime $M$. We choose a fixed global time function $t$ with its gradient a global timelike vector field in $M$ so that its lightlike hypersurface admits a global null vector field $\xi_{1}$. Now, it follows from the well know Hopf-Rinow theorem that we can choose our corresponding leaf $H^{\prime}$ a complete Riemannian hypersurface of $M$. Consequently, we have constructed a globally null manifold ( $H, h, v=$ constant) as a lightlike hypersurface of an almost metric PF-spacetime of example 3.2. A simple example is the Minkowski spacetime with $\left(H, h_{a b}=\delta_{a b}, v=\right.$ constant) its globally null hypersurface.

Just as Beem and Ehrlich [1] constructed an extended class of globally hyperbolic warped product Lorentz manifolds, to construct some more physical examples of globally null manifolds, in 2001, Duggal [6] proposed the following concept of lightlike warped product manifolds: Let $\left(H, g_{H}\right)$ and $\left(N, g_{N}\right)$ be a lightlike and a Riemannian manifold of dimensions $n_{1}$ and $n_{2}$ respectively, where the $\operatorname{RadTH}$ is of rank $r$. Let $\theta$ be a smooth function on $H, \pi: H \times N \rightarrow H$ and $\eta: H \times N \rightarrow N$ be the projection maps given by $\pi(x, q)=x$ and $\eta(x, q)=q$ for $(x, q) \in H \times N$ respectively, where the projection $\pi$ on $H$ is with respect to screen $S(T H)$.

Definition 3.1. The product manifold $M=H \times N$ is said to be a lightlike warped product $H \times_{\theta} N$, with the degenerate metric $g$ defined by

$$
g(X, Y)=g_{H}\left(\pi_{\star} X, \pi_{\star} Y\right)+(\theta \circ \pi)^{2} g_{N}\left(\eta_{\star} X, \eta_{\star} Y\right),
$$

for every $X, Y$ of $M$ and $\star$ is the symbol for the tangent map.

It follows that $\operatorname{RadTM}$ of $M$ still has rank $r$ but $\operatorname{dim}(M)=n_{1}+n_{2}$ and $\operatorname{dim}(S(T M))=n_{1}+n_{2}-r$. Consistent with above example we assume that $\left(H, g_{H}\right)$ is globally null for which $r=1$ and $\left(N, g_{N}\right)$ is a complete Riemannian manifold. Thus, we quote the following theorem. The proof is common with the proof of Lorentzian case [1]:

Theorem 3.2. (Duggal [6]). Let $\left(H, g_{H}\right)$ and $\left(N, g_{N}\right)$ be lightlike and Riemannian manifolds respectively. Then the warped product $\left(M=H \times{ }_{\theta} N, g\right)$ is globally null if and only if both the following conditions hold:

(1) $\left(H, g_{H}\right)$ is globally null.

(2) $\left(N, g_{N}\right)$ is a complete Riemannian manifold.

It is easy to show that De-Sitter, Robertson-Walker and Schwarzschild spacetimes admit globally null hypersurfces which satisfy above theorem and are physical models of null hypersurfaces of the almost metric PF-spacetime of Example 3.2.

Open problems 2.

(a) Yano [20] has proved that the existence of an $f$-structure is equivalent to the reducibility of the structure group of $T M$ to $U(m / 2) \times O(q)$, where $m$ is even. This raises following question? Find the structure group of $T M$ for the existence of an almost PF-structure of a PF-manifold $M$. The case $m$ even is a generalization of above Yano's result, subject to some conditions on the pair $(\lambda, \mu)$. The case $m$ odd seems quite different so we request input from interested researchers.

(b) As stated in open problem 1(b) of previous section on the classification of almost metric PCR-manifolds, a similar classification of almost metric PF-manifolds is needed to study the geometry of APK and PK-manifolds. (c) Blair [4] has proved that on a K-manifold the $q$ vector fields $\xi_{a}$ are Killing. It is an open question to find metric symmetry (conformal, homothetic or Killing) of these $q$-vector fields of PK-manifolds.

(d) Find integrability conditions of PF-structure tensor $f$ and its distributions.

(e) Although we have above specific examples of odd and even dimensional semi-Riemannian and lightlike metric PF-manifolds, it is an open question of an arbitrary existence (or non-existence) of semi-Riemannian metric for a PF-manifold. We only know from Blair [4] and Duggal-Sahin [8][pages 307-308] the existence of Riemannian and Lorentzian metric, respectively, for f-manifolds. 


\subsection{Contact Pseudo Framed manifolds}

Let $M$ be an $(n=m+1)$-dimensional almost metric contact pseudo framed (CPF) manifold $(M, g, f, \lambda, \eta, \xi)$ where $\eta$ is a 1 -form (called contact form), $\xi$ is a vector field, called characteristic vector field, $g$ is a semiRiemannian metric and $\mathrm{f}$ is a $(1,1))$ tensor field satisfying

$$
\begin{aligned}
f^{3}-\lambda^{2} f & =0, \quad \eta(\xi)=1, \quad \forall X \in T(M) . \\
g(f X, f Y) & =\mu\left(g(X, Y)+\epsilon \sigma^{2} \eta(X) \eta(Y)\right), \\
g(X, \xi) & =\epsilon \sigma^{2} \eta^{a}(X), \quad g(\xi, \xi)=\epsilon \sigma^{2},
\end{aligned}
$$

where $\epsilon=+1$ or -1 according as $\xi$ is spacelike or timelike, $\sigma$ and $\mu$ are real non-zero functions. Also, as per previous section there exists an almost PCR-distribution $D$ given by $\left(\bar{J}=f / D, \bar{J}^{2}=\lambda^{2} I, \eta=0\right)$. It is important to mention that in above definition $\xi$ is never a lightlike (null) vector field. We highlight that, contrary to the Riemannian contact manifolds, we have following subcases of CPF-manifolds.

Riemannian CPF-manifolds: In Example 3.1 if we consider a 4-dimensional Riemanian manifold $(M, g)$ with $\epsilon=1$ and $q=1$, then, it is easy to see that $(M, g)$ is an example of even dimensional almost metric CPFmanifold. Furthermore, this example can be extended to $\operatorname{dim}(M)=2 n$, with $\operatorname{dim}(D)=2 n-1$ and $q=1$ to show that there do exist even dimensional almost metric Riemannian PC-manifolds. Using this example, we suggest further study on even dimensional almost metric Riemannian CPF-manifolds discussing the similarities and differences compared with the results of odd dimensional almost contact Riemannian manifolds. On the other hand, as per open problems 1(a), we do not know the existence of even dimensional spacelike PCRdistributions $D$ needed to construct an example of odd dimensional almost metric CPF-manifold which admits a 2 -form $\Omega$.

Lorentzian CPF-manifolds: Following are two cases for this type of manifolds:

Case 1. $\epsilon=-1$ and $D$ spacelike. Case 2. $\epsilon=1$ and $D$ timelike. For both these cases we follow the terminology used in [5] and say that the corresponding almost CPF-manifold is an almost Lorentzian (in particular, spacetime) CPF-manifold. Following are physical models of these two cases:

Physical Model(case 1). Let $\left(\bar{M}^{m}, \bar{g}, \bar{J}^{2}=\lambda^{2} I\right)$ be an almost metric Riemannian PCD-manifold with $\bar{g}$ compatible with $\bar{J}$ if $\bar{g}(J \bar{X}, J \bar{Y})=\mu \bar{g}(\bar{X}, \bar{Y})$, for every vector fields $\bar{X}, \bar{Y}$ and some non-zero function $\mu$ of $\bar{M}$. As a particular case of Mathematical Model of this section, we construct a product manifold defined by $M=\left[(\bar{M} \times R), g=\bar{g}-d t^{2}\right]$. Let $X=\left(\bar{X}, \eta(X) \frac{d}{d t}\right)$ be a vector field on $M$ where $\bar{X}$ is tangent to $\bar{M}, t$ is a coordinate of $R$ and $\eta(X)$ is a smooth function on $M$. Set $\eta=d t$ so that $\xi=\left(0, \frac{d}{d t}\right)$ is a vector field tangent to $M$. Suppose $\mathrm{f}$ is a $(1,1)$ tensor field on $M$ defined by

$$
f(X)=f\left(\bar{X}, \eta(X) \frac{d}{d t}\right)=(\bar{J} \bar{X}, 0) .
$$

Then, we obtain

$$
\begin{aligned}
& f^{2}(X)=\lambda^{2} X+\eta(X) \xi, \quad f(\xi)=0, \quad \eta f=0, \quad \eta(\xi)=1, \\
& g(\xi, \xi)=-1, \quad g(f X, f Y)=\mu(g(X, Y)-\eta(X) \eta(Y)),
\end{aligned}
$$

which shows that $(M, g, f, \lambda, \mu)$ is almost metric a Lorentzian CPF-manifold, with a timelike characteristic vector field $\xi$.

Example 3.3. In Example 3.1 we set $\epsilon=1, \quad q=1, \quad g\left(\xi_{4}, \xi_{4}\right)=g(\xi, \xi)=-1$. Then, it is easy to see that $(M, g, \eta, \xi)$ is an almost metric Lorentzian CPF-manifold. In particular, if $\xi$ is global then $M$ is a spacetime. Following the construction of physical examples of previous section, it is straightforward to show that 4dimensional (in general $2 n$-dimensional) de-Sitter and Robertson-Walker spacetimes are examples of physical model(class 1) of CPF-manifolds.

Physical Model(case 2). Let $\left(\bar{M}^{m}, \bar{g}, \bar{J}^{2}=\lambda^{2} I\right)$ be an almost metric Lorentzian PCD-manifold with $\bar{g}$ compatible with $\bar{J}$ if $\bar{g}(J \bar{X}, J \bar{Y})=\mu \bar{g}(\bar{X}, \bar{Y})$, for every vector field $\bar{X}, \bar{Y}$. Construct a product manifold defined by $\left.M=(\bar{M} \times R), g=\bar{g}+d u^{2}\right)$, where $u$ is coordinate of $R$ and $\eta(X)$ is a smooth function on $M$. Let $X=\left(\bar{X}, \eta(X) \frac{d}{d u}\right)$ be a vector field on $M$ where $\bar{X}$ is tangent to $\bar{M}$. Set $\eta=d u$ so that $\xi=\left(0, \frac{d}{d u}\right)$ is a vector field tangent to $M$. Let $\xi$ be a spacelike vector field tangent to $M$. Proceeding as in case 1 , we say that $(M, g, f, \lambda, \mu)$ is an almost metric Lorentzian CPF-manifold, with a spacelike characteristic vector field $\xi$.

Example 3.4. In Example 3.1 if we consider a 4-dimensional Lorentzian manifold $(M, g)$ with $\epsilon=-1$ and $q=1$ , then, it is easy to see that $(M, g)$ is an example of even dimensional almost metric CPF-manifold. This example 
can be extended to $\operatorname{dim}(M)=2 n$, with $\operatorname{dim}(D)=2 n-1$. Here also one can construct examples of de-Sitter and Robertson-Walker CPF-spacetimes.

Lightlike CPF-manifolds. For this case we need following example.

Example 3.5. Suppose $\left\{\xi_{1}, \xi_{2}, \xi_{3}, \xi_{4}\right\}$ is an adapted basis for $T_{p} M$ at a point $p$ of a $(3+1)$-dimensional manifold $(M, g)$ endowed with a real tensor field $f$ of type $(1,1)$ and two real non-zero function $\lambda$ and $\mu$ on $M$. Assume $M$ admits a 2-form $\Omega$ defined by (2.5). Then, as per Proposition 2.1, $\mu=-\lambda^{2}$. Let $f \xi_{1}=\xi_{1}$. Using this and the metric compatible equation (2.2) we get $g\left(f \xi_{1}, f \xi_{1}\right)=-\lambda^{2} g\left(\xi_{1}, \xi_{1}\right)=\lambda^{2} g\left(\xi_{1}, \xi_{1}\right)$. This implies that $\bar{g}\left(\xi_{1}, \xi_{1}\right)=0$ so $\xi_{1}$ is a null vector. Let $g\left(\xi_{a}, \xi_{a}\right)=\sigma_{a}^{2}$ for each $\left.a=2,3,4\right)$, where each $\sigma_{a}$ is a real function on $M$. Suppose $f \xi_{2}=\xi_{3}, \quad f \xi_{3}=\lambda^{2} \xi_{2}, \quad f \xi_{4}=0$. Then, following Example 3.1 and taking $X=\Sigma_{a} \eta^{a}(X) \xi_{a}$ we get

$$
g(X, Y)=\sigma_{2}^{2}\left(\eta^{2}(X) \eta^{2}(Y)-\lambda^{2} \eta^{3}(X) \eta^{3}(Y)\right)+\sigma_{4}^{2} \eta^{4}(X) \eta^{4}(Y), \quad \forall \bar{X}, \bar{Y} \in \Gamma(T M) .
$$

Thus, as explained in section 2, $(M, g, f, \lambda)$ is a 1-lightlike almost metric CPF-manifold which admits a 1dimensional radical distribution $\operatorname{RadT} \bar{M}=\left\{\xi_{1}\right\}$ and 3-dimensional screen distribution $S(T M)=\left\{\xi_{2}, \xi_{3}, \xi_{4}\right\}$ such that

$$
\begin{aligned}
T M & =\operatorname{Rad}(T M) \oplus_{\text {orth }} S(T M) \oplus\left\{\xi_{4}\right\}, \quad \operatorname{rank}(\bar{g})=2, \\
f X & =\lambda \eta^{1}(X) \xi_{1}+\eta^{2}(X) \xi_{3}+\lambda^{2} \eta^{3}(X) \xi_{2} \\
f^{3} & =\lambda^{2} f . \quad f \operatorname{RadTM}=\operatorname{RadTM}, \quad[X, Y] \in \operatorname{RadTM}, \quad \forall X, Y \in \operatorname{RadTM} .
\end{aligned}
$$

Using Examples $2.3=2.4$, one can show that there exist $r$-lightlike almost metric CPF-manifolds which admit a 2 -form $\Omega$. As stated in Section 1, in a followup paper we plan to discuss more physical examples of lightlike CPF-hypersurfaces.

Remark 3.1. In this paper we have focused on a variety of examples (with physical interpretation) to justify, for the first time in the literature, the existence of odd dimensional Riemannian, Lorentzian (in particular, spacetime) and $r$-lightlike almost metric (PCR) and even dimensional (CPF) manifolds compared with the even dimensional $\mathrm{CR}$ and odd dimensional contact manifolds, respectively. We have also shown that the metric of our new definitions of PCR and PF manifolds is not severely restricted. This initial paper opens a new area of research on the geometry of odd dimensional PCR-manifolds, even dimensional CPF-manifolds and their submanifolds with applications. Since we have proposed several open problems in this paper, an input from interested readers is requested before proceeding with further study on the geometry and physics of PCR and $\mathrm{PF}$ (in particular, $\mathrm{CPF}$ ) manifolds and their submanifolds.

\section{References}

[1] Beem, J. K., Ehrlich, P. E.: Global Lorentzian Geometry. Marcel Dekker Inc. New York (1981). Second Edition (with Easley, K. L.) (1996).

[2] Bejancu, A.: Geometry of CR-submanifolds. D. Reidel Publishing Company. Boston (1986).

[3] Blair, D. E.: Contact manifolds in Riemannian geometry. Lecture notes in Math. Springer-Verlag. Berlin (1976).

[4] Blair, D. E.: Geometry of manifolds with structure group $U(n) \times O(s)$. J. Diff. Geometry 4, 155-167 (1970).

[5] Duggal, K. L.: Spacetime manifolds and contact structures. Int. J. Math. \& Math. Sci. 13, 545-554 (1990).

[6] Duggal K. L.: Warped product of lightlike manifolds. Nonlinear Anal. 47, 3061-3072 (2001).

[7] Duggal, K. L., Jin, D. H.: Null Curves and Hypersurfaces of Semi-Riemannian Manifolds. World Scientific (2007).

[8] Duggal, K. L., Sahin, B.: Differential geometry of lightlike submanifolds. Birkhäuser (2010).

[9] Duggal, K. L., Sharma, R.: Symmetries of spacetimes and Riemannian manifolds. Kluwer Academic Publishers (1999).

[10] Eliopoulos, H.: On the general theory of differentiable manifolds with almost tangent structure. Canad. Math. Bull. 8, 721-748 (1965).

[11] Flaherty, E. T.: Hermitian and Kählerian Geometry in Relativity. Lecture Notes in Physics. Springer-Verlag. Berlin (1976).

[12] Gray, A., Hervella, L. M.: The sixteen classes of almost Hermitian manifolds. Ann. Mt. Pura Appli. 123, 35-58 (1980).

[13] Kaehler, E.: Uber eine bemerkenswerte Hermitische metrik. Abh. Math. Sem. Hamburg 9, 173-186 (1933).

[14] Legrand, G.: Sur les variéteś à structure de presque produit complexe. C.R. Acad. Sci. Paris 242, 335-337 (1956).

[15] Middleton. C. A., Stanley. E.: Anisotropic evolution of 5D Friedmann-Robertson-Walker spacetime. Phys. Rev. D84 085013, (2011).

[16] Newlander, A., Nirenberg, L.: Complex analytic coordinates in almost complex manifolds. Ann. Math. 65, 391-404 (1957).

[17] O'Neill, B.: Semi-Riemannian Geometry with Applications to Relativity. Academic Press. New York (1983).

[18] Penrose, R.: Physical spacetime and non realizable CR-structure. Proc. of symposia in Pure Math. 39, 401-422 (1983).

[19] Walker, A. G.: Completely symmetric spaces. J. Lond. Math. Soc. 19, 219-226 (1944).

[20] Yano, K.: On a structure defined by a tensor field of type $(1,1)$ satisfying $f^{3}+f=0$. Tensor N.S. 14, 99-109 (1963). 


\section{Affiliations}

KRISHAN LAL DUGGAL

AdDress: Department Of Mathematics And Statistics, University Of Windsor, Windsor, Ontario, N9b3p4, Canada.

E-MAIL: yq8@uwindsor.ca

ORCID ID: 0000-0003-2967-2727 